\title{
Tissue-specific regulation of cyclin E transcription during Drosophila
}

\section{melanogaster embryogenesis}

\author{
Lynn Jones*, Helena Richardson‡ and Robert Saint ${ }^{\ddagger}$ \\ Centre for the Molecular Genetics of Development and Department of Molecular Biosciences, University of Adelaide, \\ South Australia, 5005, Australia \\ *Present address: Department of Genetics, Yale University School of Medicine, New Haven, CT 06510, USA \\ ¥Authors for correspondence (e-mail: robert.saint@adelaide.edu.au) \\ Accepted 11 August; published on WWW 9 October 2000
}

\section{SUMMARY}

Cyclin $E$ is an essential regulator of $S$ phase entry. We have previously shown that transcriptional regulation of the gene that encodes Drosophila cyclin E, DmcycE, plays an important role in the control of the $G_{1}$ to $S$ phase transition during development. We report here the first comprehensive analysis of the transcriptional regulation of a $G_{1}$ phase cell cycle regulatory gene during embryogenesis. Analysis of deficiencies, a genomic transformant and reporter gene constructs revealed that $D m c y c E$ transcription is controlled by a large and complex cis-regulatory region containing tissue- and stagespecific components. Separate regulatory elements for transcription in epidermal cells during cell cycles 14-16, central nervous system cells and peripheral nervous system cells were found. An additional cis-regulatory element drives transcription in thoracic epidermal cells that undergo a 17th cell cycle when other epidermal cells have arrested in $G_{1}$ phase prior to terminal differentiation. The complexity of $D m c y c E$ transcriptional regulation argues against a model in which $D m c y c E$ transcription is regulated simply and solely by $G_{1}$ to $S$ phase transcription regulators such as RB, E2F and DP. Rather, our study demonstrates that tissue-specific transcriptional regulatory mechanisms are important components of the control of cyclin $E$ transcription and thus of cell proliferation in metazoans.

Key words: cyclin E, Cell cycle, Transcriptional regulation, Drosophila, Development, S phase

\section{INTRODUCTION}

In recent years, knowledge of the regulatory molecules that control the eukaryotic cell cycle has grown dramatically. Two protein families, Cyclin-dependent serine/threonine kinases (Cdks) and cyclins, are central components of cell cycle regulation (reviewed in Pines and Hunter, 1991; Reed, 1992; Follette and O'Farrell, 1997). The $\mathrm{G}_{1}$ cyclin, Cyclin E, was shown to be essential and rate limiting for progression into $\mathrm{S}$ phase in Drosophila embryonic and eye imaginal disc cells (Knoblich et al., 1994; Richardson et al., 1995) and also in mammalian cells (Ohtsubo, and Roberts, 1993; Resnitzky et al., 1994; Ohtsubo et al., 1995). We have previously shown that transcriptional regulation of the Drosophila cyclin $E$ gene (DmcycE) is an important component in the control of cell proliferation during Drosophila development. The cycle $17 \mathrm{G}_{1}$ arrest that occurs in the embryonic epidermal cells prior to differentiation and the transient $\mathrm{G}_{1}$ arrest of cells in the morphogenetic furrow of the developing eye disc both require downregulation of DmcycE (Knoblich et al., 1994; Richardson et al., 1995).

Multicellular development requires the coordination of cell division with morphogenic and developmental processes, suggesting that cell cycle progression must ultimately be controlled by developmental regulatory mechanisms. Given that different tissues exhibit widely variable cell cycle kinetics and that different regulators such as Dpp, Wingless, Notch and Prospero are responsible for proliferation or cell cycle arrest in different developmental contexts (Masucci et al., 1990; Skaer and Martinez-Arias, 1992; Horsfield et al., 1998; Go et al., 1998; Richter et al., 1998; Johnston and Edgar, 1998; Li and Vaessin, 2000; reviewed by Serrano and O'Farrell, 1997; Myster and Duronio, 2000), it will be important to determine the nature of the links between developmental regulators and cell cycle control if we are to understand the cell proliferation aspects of morphogenesis.

One mechanism by which developmental signals and cues could regulate cell proliferation is transcriptional control of cell cycle regulatory genes. DmcycE is a cell cycle regulatory gene that could be subject to this method of control, in order to link $\mathrm{S}$ phase with developmental processes during Drosophila development. The pattern of zygotic DmcycE transcription during embryonic development is complex and can be divided into three distinct modes, based on the type of modified cell cycle that is observed during embryogenesis.

Zygotic DmcycE transcription is first observed ubiquitously, at low levels, throughout the epidermis during mitotic cycles 14-16 (Richardson et al., 1993), although all cycles prior to cycle 17 can occur without zygotic transcription (Knoblich et al., 1994), presumably due to the presence of maternally 
derived products. These embryonic cycles consist of a $G_{2}$ phase of variable length, mitosis and an $\mathrm{S}$ phase that initiates without an intervening $G_{1}$ phase (Edgar and O'Farrell, 1990). Cell division during these cycles occurs in a complex spatiotemporal pattern with groups of cells, termed mitotic domains, undergoing mitosis synchronously (Foe, 1989). The pattern of $\mathrm{S}$ phases, as monitored by bromodeoxyuridine (BrdU) incorporation, is complex, as a result of the preceding mitoses. During this period, S phases do not correlate with the ubiquitous epidermal DmcycE transcription (Richardson et al., 1993). A similar mode of regulation also appears to operate in the proliferating cells of the embryonic central and peripheral nervous systems (CNS and PNS) where cycles lack detectable $\mathrm{G}_{1}$ phases (Hartenstein et al., 1987; Edgar and O'Farrell, 1990; Weigmann and Lehner, 1995). Transcription of DmcycE is then downregulated in most cells by stage 11 , close to and perhaps at the time that cell division ceases after mitosis 16 (Richardson et al., 1993; Knoblich et al., 1994). Downregulation of DmcycE expression at this developmental stage is essential for the $G_{1}$ arrest observed in the epidermis prior to differentiation (Knoblich et al., 1994).

A second mode of DmcycE transcription is observed in a subset of epidermal cells in the lateral thoracic region (thoracic segments 1, 2 and 3) of the embryo. These cells, here termed the epidermal thoracic patches, complete a 17th mitotic cell cycle (Bate and Martinez-Arias, 1991; Knoblich et al., 1994) and are different to the remainder of the epidermal cells, which arrest in $\mathrm{G}_{1}$ phase of cell cycle 17 . In zygotic null DmcycE mutants, where maternal sources of Cyclin E become depleted after cycle 16, the epidermal thoracic patch cells arrest in $\mathrm{G}_{1}$ phase and do not enter the 17 th $\mathrm{S}$ phase (Knoblich et al., 1994), indicating that zygotic DmcycE transcription is essential for $\mathrm{S}$ phase progression in these cells. In addition, ectopic expression of DmcycE induces all dorsal epidermal cells, including the epidermal thoracic patch cells, to coordinately enter $\mathrm{S}$ phase, indicating that $D m c y c E$ transcription is limiting for entry of these cells into $S$ phase (Knoblich et al., 1994). $D m c y c E$ transcription is downregulated coordinately in all epidermal cells, but is activated specifically in the cells of the thoracic patches in a pattern that precedes the 17 th $\mathrm{S}$ phase (Knoblich et al., 1994). Thus the cycle 17 epidermal thoracic patch cells are the first cells to exhibit $G_{1}, S, G_{2}$ and $M$ phases and are the first embryonic cells that are regulated at the $G_{1}$ to $S$ phase transition by transcriptional regulation of DmcycE.

The third mode of DmcycE transcription is observed in endoreplicating tissues such as the gut, where the cell cycle consists of $\mathrm{S}$ phases with intervening gap phases but no mitoses (Smith and Orr-Weaver, 1991). DmcycE transcription in these tissues is spatially regulated and coincides with $S$ phase (Knoblich et al., 1994). The cycling of DmcycE levels in endoreplicating cells is essential for the rounds of $\mathrm{S}$ phases observed in these cells (Follette et al., 1998; Weiss et al., 1998). These tissues are further examples of embryonic cells that have regulated $G_{1}$ to $S$ phase transitions that are dependent on DimcycE transcription.

Developmental cues are implicated in the regulation of DmcycE transcription in Drosophila. For example, in string (stg) mutant embryos, DmcycE is transcribed in the correct spatiotemporal pattern and is downregulated at the time when cycle $17 \mathrm{G}_{1}$ phase arrest would normally occur, despite the fact that all cells remain in $\mathrm{G}_{2}$ phase of cycle 14 (Knoblich et al.,
1994). Thus in these cells, DmcycE transcription is independent of cell cycle progression and must be responding to developmental cues.

To investigate the relationship between embryonic development and cyclin $E$ transcriptional control, we have dissected the DmcycE transcriptional regulatory region using a combination of DmcycE deficiencies, a genomic transformant and reporter gene constructs. In this first comprehensive analysis of developmental transcriptional regulation of a key $\mathrm{G}_{1}$ phase cell cycle regulatory factor, we show that $D m c y c E$ has a large regulatory region containing multiple tissue-specific cis-acting sequences that act to coordinate the $\mathrm{G}_{1}$ to $\mathrm{S}$ phase transition with embryonic development.

\section{MATERIALS AND METHODS}

\section{Generation of constructs for germline transformation and generation of transgenic flies}

The regions of genomic DNA used in all constructs are summarised in Fig. 1. The construct used to attempt genomic rescue consists of a $10.7 \mathrm{~kb} K p n \mathrm{I}$ genomic fragment covering the DmcycE type I transcript cloned into $P\{$ CaSpeR -4$\}$ to generate $P\left[w^{+}\right] T I$. Transgenic flies containing $P\left[w^{+}\right] T I$ were obtained by $P$-element-mediated germline transformation of $w^{1118}$ embryos and selection of $w^{+}$transgenic flies. Three independent homozygous viable transformant lines were obtained and used in further analysis.

The 1.OTATAlacZ DmcycE promoter-lacZ reporter construct (see Fig. 1) was made by cloning the $1.0 \mathrm{~kb}$ genomic HindIII/EcoRI fragment upstream of a minimal $h s p 27$ promoter and a nuclear targeted lacZ (nlacZ) reporter gene (from G. Riddihough). The assembled DNA was then cloned into the $P\{C a S p e R-4\}$ transformation vector and used to transform the germline of flies using $P$-element-mediated transformation.

All remaining reporter gene constructs utilised the DmcycE promoter in place of the $h s p 27$ promoter. An NcoI site was created at the $D m c y c E$ ATG by site-directed mutagenesis. The resulting $2.9 \mathrm{~kb}$ $N c o$ I fragment containing the DmcycE Type I 5' UTR and proximal promoter sequences was ligated to the $N c o$ I site corresponding to the ATG translation start site of nlacZ in a pBluescriptII (Stratagene) vector to create $p B S T-2.9 l a c Z$. Constructs used in this study were derived from $p B S T-2.9 l a c Z$ by sequential addition of DmcycE genomic fragments. Most of the endogenous promoter constructs contained contiguous upstream $D m c y c E$ genomic sequences from the Type I DmcycE ATG, the $3.3+1.9 l a c Z$ construct being the only noncontiguous endogenous promoter construct characterised in this study. Constructs were assembled in pBluescriptII (Stratagene), excised using KpnI and NotI, cloned into the P\{CaSpeR-4\} transformation vector and transgenic flies generated as described above.

\section{Whole-mount RNA in situ hybridisation, bromodeoxyuridine (BrdU) incorporation and immunohistochemistry}

Whole-mount RNA in situ hybridisation to embryos was performed essentially as described by Tautz and Pfeifle (1989). For DmcycE RNA in situ hybridisation, lacZ expression from either a $C y \mathrm{OwglacZ}$ or a $C y \mathrm{O} f t z l a c Z$ balancer chromosome was detected by antibody staining to allow unambiguous identification of the homozygous DmcycE mutant embryos. For BrdU labelling to detect $\mathrm{S}$ phases, embryos were labelled for 30 minutes with BrdU as previously described by Richardson et al. (1993). After labelling and fixation of $D m c y c E$ mutations, lacZ expression from either the $C y O w g l a c Z$ or a CyOftzlacZ balancer chromosome was used to identify homozygous mutant embryos, as described above.

All embryos were mounted in $80 \%$ glycerol in $1 \mathrm{x}$ PBS and visualised and photographed on a Zeiss Axiophot microscope using Nomarski optics. 


\section{RESULTS}

\section{Zygotic DmcycE transcription is controlled by a large cis-regulatory region}

The DmcycE locus produces two transcripts, Type I and Type II, from different promoters (Richardson et al., 1993; L. Jones, M. Silson, W. Winnall, R. Saint and H. E. Richardson, unpublished). The Type I promoter drives embryonic zygotic transcription, while the Type II promoter is used to generate the maternally derived transcripts present in the embryo (Richardson et al., 1993; D. Crack, J. Secombe, A. Brumby, M. Coombe, R. Saint and H. E. Richardson, unpublished). Because of its role in embryonic zygotic DmcycE expression, the studies described here focus on the regulation of DmcycE Type I transcription. The extent of the cis-acting regulatory region controlling zygotic DmcycE (Type I) transcription was initially investigated by generating transgenic flies carrying a $10.7 \mathrm{~kb} \mathrm{KpnI} \mathrm{genomic} \mathrm{DNA} \mathrm{fragment} \mathrm{that} \mathrm{spanned} \mathrm{the} \mathrm{zygotic}$ $D m c y c E$ transcript $\left(P\left[w^{+} T I\right]\right)$ and included approximately 4.8 $\mathrm{kb}$ upstream and $1.8 \mathrm{~kb}$ downstream of this transcript (Fig. 1). Two independent transformants homozygous for $\mathrm{P}\left[\mathrm{w}^{+} \mathrm{TI}\right]$ failed to rescue to viability a DmcycE null allele $\left(D m c y c E^{A R 95}\right)$ trans-heterozygous with either $D f(2 L) T E 35 D$ 17 or $D f(2 L) T E 35 D-19$, two deficiencies that uncover the DmcycE gene (data not shown; Knoblich et al., 1994).

To determine if the $P\left[w^{+} T I\right]$

transgene expressed the zygotic DmcycE transcript during embryogenesis, whole-mount in situ hybridisation was carried out on embryos homozygous for each of three independent lines of the transgene in a homozygous DmcycE deficiency background. In the absence of the transgene, no transcripts were detected in post-blastoderm embryos homozygous for either of the two DmcycE deficiencies $D f(2 L) T E 35 D$ 19 or $D f(2 L) T E 35 D-17$ (Fig. $2 \mathrm{C}, \mathrm{H}$ and results not shown). In embryos homozygous for a $D m c y c E$ deficiency and carrying the $P\left[w^{+} T I\right]$ transgene, $D m c y c E$ transcripts were detected in epidermal tissues during mitotic cycles 14-16 (data not shown) and in the proliferating CNS (Fig. 2B) in a pattern similar to that observed in $D_{\text {DmcycE }}{ }^{+}$sibling embryos, which express DmcycE in a wild-type pattern (Fig. 2A). The proliferating PNS cells of $P\left[w^{+} T I\right]$ transgenic, DmcycE deficient homozygous embryos also showed DmcycE expression in a pattern similar to $D m c y c E^{+}$siblings, with the exception of PNS neuroblasts in the maxillary and labial head segments, in which $D m c y c E$ transcripts were reduced or absent (Fig. 2B, compare with A). In addition, DmcycE transcripts from the transgene were not detectable in the epidermal thoracic patches (Fig. 2B, $\mathrm{B}^{\prime}$, compare with A, $\mathrm{A}^{\prime}$ ). The absence of DmcycE expression in the epidermal thoracic patches correlates with the absence of BrdU incorporation into these cells in DmcycE deficient embryos carrying the $P\left[w^{+} T I\right]$ transgene (Fig. 2E, compare with D). Finally, staining in the endoreplicating gut (Fig. 2F) was not detectable above the background observed using a cyclin $E$ probe with cyclin $E$ deficient embryos (Fig. 2G, compare with H). In $P\left[w^{+} T I\right]$ transgenic DmcycE deficient embryos, expression of DmcycE correlated with $\mathrm{S}$ phases in all tissues, as revealed by BrdU incorporation (Fig. 2E and data not shown). These data indicate that the $10.7 \mathrm{~kb} K p n$ I genomic fragment could drive DmcycE expression at sufficiently high levels, in the tissues in which it is expressed, to induce entry into $\mathrm{S}$ phase.

These results demonstrate that the regions covering and closely flanking the zygotic Type I transcript in the $P\left[w^{+} T I\right]$ transgene contain cis-acting regulatory sequences that drive $D m c y c E$ transcription in the constitutive epidermal pattern during mitotic cycles 14-16, in the proliferating CNS and most of the proliferating PNS. However this pattern of DmcycE expression represents only part of the wild-type pattern seen during embryogenesis. The lack of DmcycE transcripts and $\mathrm{S}$ phases in the epidermal thoracic patches and a subset of the proliferating PNS cells shows that part or all of the cis-acting sequences regulating DmcycE transcription in these tissues are

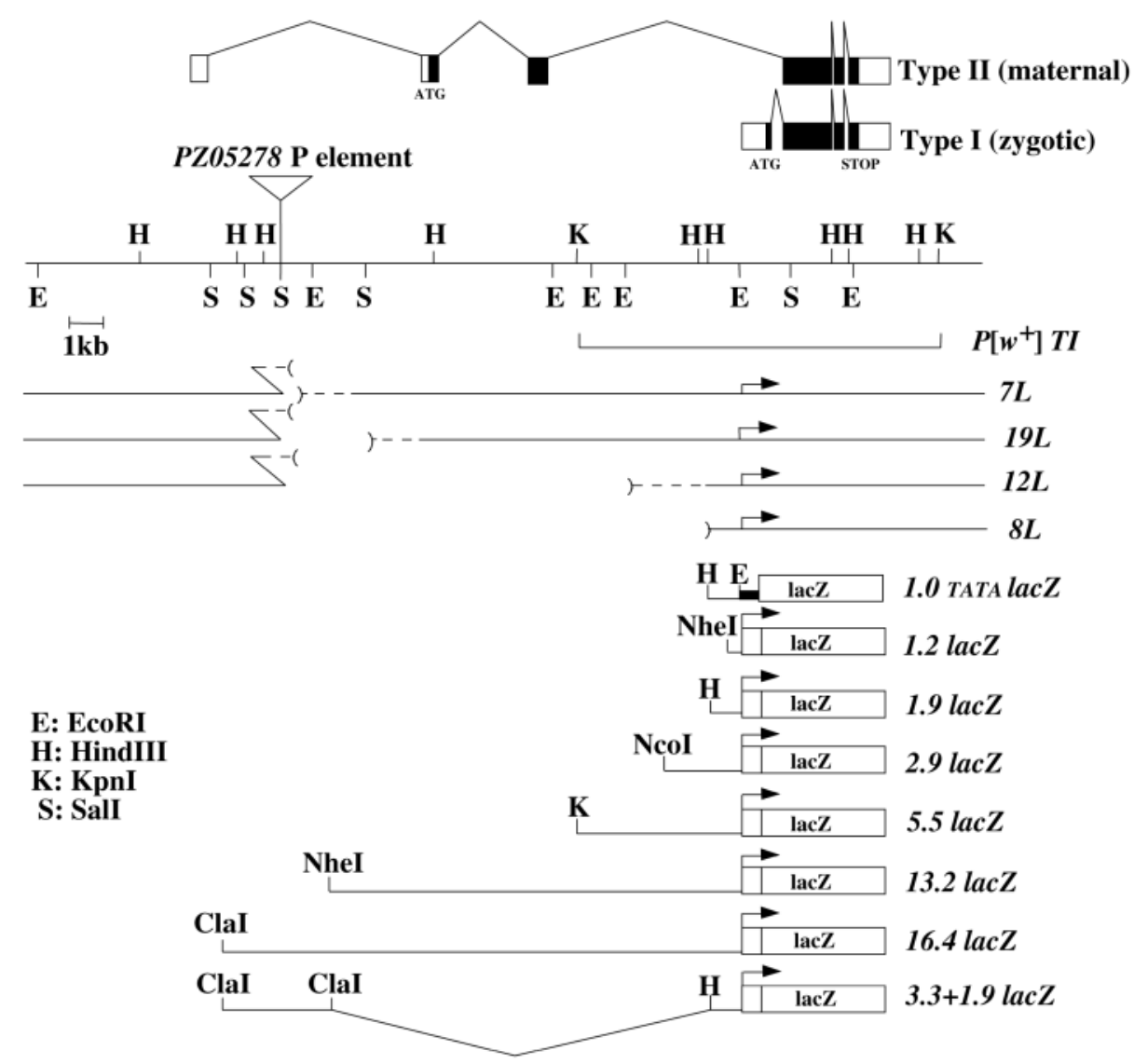

Fig. 1. Deficiencies and transformation constructs used in this study: a summary of the deletions and constructs used. The $T I$ genomic fragment used in the rescue attempt, a series of P-element excision-derived deficiencies and the genomic fragments used to generate a series of reporter gene constructs are shown in relation to a genomic map of DmcycE. The broken lines indicate uncertainties in the position of deficiency breakpoints. 

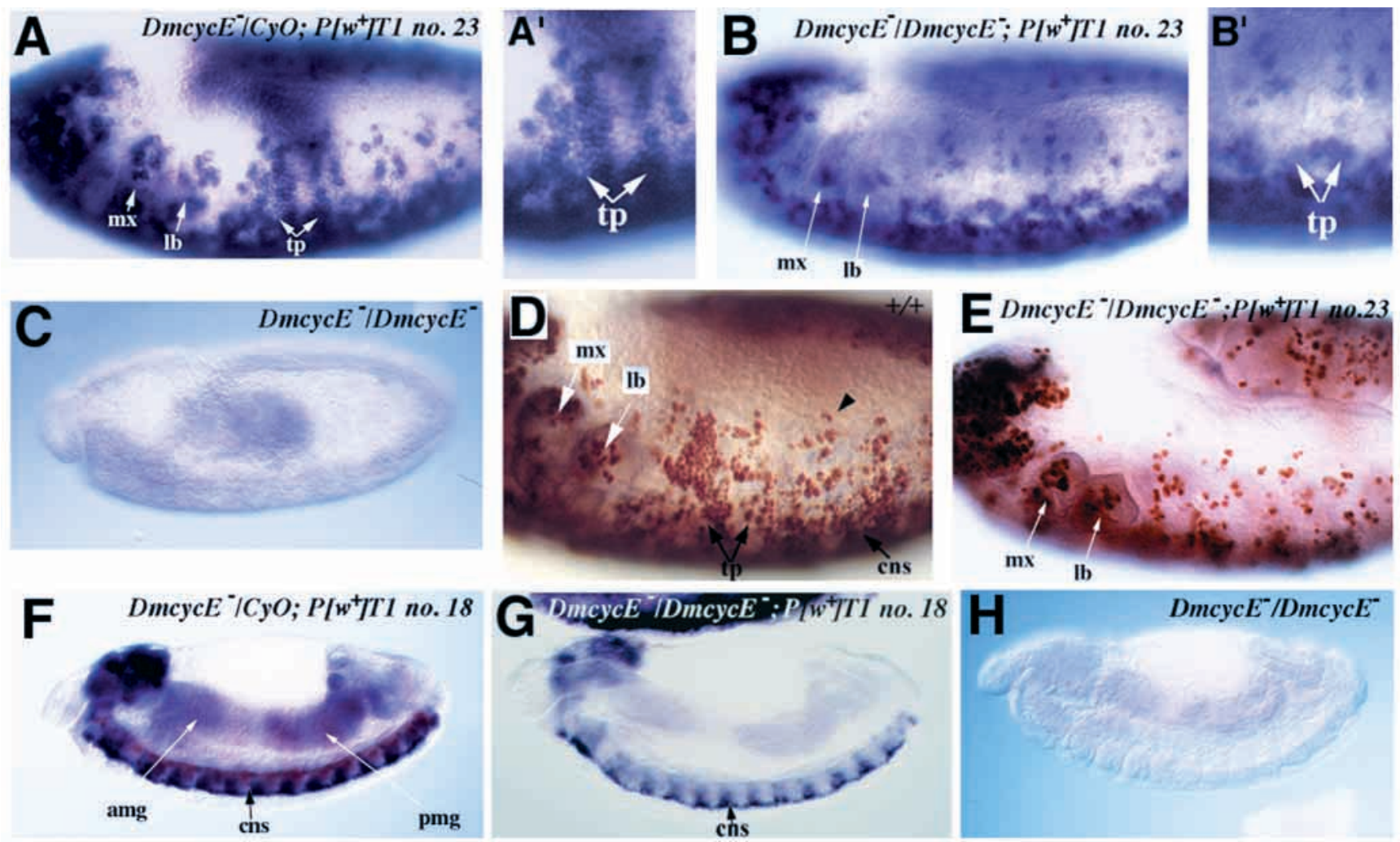

Fig. 2. The $10.7 \mathrm{~kb} D m c y c E$ genomic transgene does not generate a complete pattern of DmcycE transcripts during embryogenesis. Wholemount in situ hybridisation using a $D m c y c E$ probe $(\mathrm{A}-\mathrm{C}, \mathrm{F}-\mathrm{H})$, BrdU incorporation (D,E). (A) A stage-11 embryo heterozygous for a $D m c y c E$ deficiency and carrying the transgene, showing normal transcript levels in the CNS, PNS and epidermal thoracic patches. (A') A higher magnification of thoracic patch expression in A, showing the epithelial nature of the cells expressing DmcycE. Out-of-focus CNS and PNS cells lie underneath these cells. (B) A stage-11 embryo homozygous for a DmcycE deficiency and carrying the $T I$ transgene, showing transcripts in the CNS and PNS, but absence of transcripts in the epidermal thoracic patches and reduced levels of transcripts in the maxillary and labial neuroblasts. (B') A higher magnification of the region that normally shows thoracic patch expression. Expression in CNS and PNS cells remains. (C) A stage-11 embryo homozygous for a DmcycE deficiency, showing absence of $c y c l i n E$ transcripts. (D) A late stage-11/early stage-12 wild-type embryo showing incorporation of BrdU into cells of the thoracic patches and CNS and PNS cells. The arrowhead indicates an example of a PNS cell that has incorporated BrdU. (E) A late stage-11/early stage-12 embryo homozygous for a $D m c y c E$ deficiency and carrying the TI transgene, showing S phases in the CNS and PNS, but not in the epidermal thoracic patches. (F) A stage-13 embryo heterozygous for a DmcycE deficiency and carrying the transgene, showing normal transcription in the CNS and endoreplicating gut. The brown stain in this figure is the result of immunohistochemical staining of $\beta$-galactosidase derived from the balancer chromosome. (G) A stage13 embryo homozygous for a DmcycE deficiency and carrying the $T I$ transgene, showing transcripts in the CNS but no staining above background in the endoreplicating gut. $(\mathrm{H})$ A stage-13 homozygous DmcycE deficiency control embryo. cns, central nervous system staining; $\mathrm{mx}$, maxillary staining; lb, labial PNS staining; tp, thoracic patches.

located outside of the $10.7 \mathrm{~kb} K p n I$ region contained in the $P\left[w^{+} T I\right]$ transgene. The lack of these regulatory elements provides an explanation for the inability of this transgene to rescue a $D m c y c E$ null mutation. Significantly, these results indicate that regulation of DmcycE transcription during embryogenesis is driven by separable tissue-specific cis-acting regulatory regions.

\section{Identification of cis-acting sequences required for $D m c y c E$ transcription in the epidermal thoracic patches and the PNS}

Three large $P$-element-mediated deletions $D m c y c E^{8 L}$, $D m c y c E^{12 L}$ and DmcycE $E^{19 L}$ (see summary in Fig. 1), which remove putative cis-acting regulatory sequences important for zygotic DmcycE transcription during embryogenesis, have been generated from a lethal $P$-element insertion allele of $D m c y c E$ $(l(2) 05278)$ that appears to specifically disrupt endoreplication
S phases (L. Jones, M. Silson, W. Winnall, R. Saint and H. E. Richardson, unpublished). Whole-mount in situ hybridisation analysis of $D m c y c E^{8 L}$ homozygous mutant embryos (the largest $P$-element-induced deletion; Fig. 1) revealed the presence of $D m c y c E$ transcripts in the epidermis and the proliferating CNS in a pattern similar to wild type, although the overall level of transcripts detected was reduced when compared with DmcycE ${ }^{+}$sibling embryos (Fig. 3B, compare with A). However, transcripts were not detected in the proliferating PNS, nor in the epidermal thoracic patches (Fig. 3B). A similar result was obtained with homozygous embryos of the second largest deficiency, Dmcyc $E^{12 L}$, except that transcripts in the epidermis and CNS were at wild-type levels (Fig. 3D, compare with C). $D m c y c E$ transcripts were also present in one or two PNS cells per parasegment in this deficiency, but were not detected in the thoracic patches (Fig. 3C,D and results not shown). BrdU incorporation revealed that the distribution of $\mathrm{S}$ phases mirrored 
Fig. 3. Phenotypic analysis of the $8 L, 12 L$ and $19 L$ deficiencies during embryogenesis. Whole-mount in situ hybridisation using a DmcycE probe (A-D, G, $\left.\mathrm{G}^{\prime}, \mathrm{H}, \mathrm{H}^{\prime}, \mathrm{I}, \mathrm{J}\right)$ and BrdU incorporation (E,F). Homozygous embryos were detected by the absence of ftzlac $Z$ expression, in the case of $\mathrm{B}, \mathrm{D}, \mathrm{H}, \mathrm{J}$, and by the absence of $w$ glac $\mathrm{Z}$ staining in F. (A-F) Stage-11 embryos. (A) DmcycE $E^{+}$sibling of the $D m c y c E^{8 L}$ embryo in $\mathrm{B}$, showing the wildtype pattern of transcripts in the CNS, PNS and epidermal thoracic patches. (B) Homozygous $D m c y c E^{8 L}$ embryo showing very reduced levels of transcripts in the CNS and absence of transcripts in the PNS and epidermal thoracic patches.

(C) $D m c y c E^{+}$sibling of the $D m c y c E^{12 L}$ embryo in $D$ showing the wild-type pattern of transcripts in the CNS, PNS and epidermal thoracic patches. (D) Homozygous Dmcyc $E^{12 L}$ embryo showing transcripts in the CNS, but very reduced PNS expression and no epidermal thoracic patches transcripts. (E) BrdU incorporation in a heterozygous DmcycE $E^{I 2 L}$ embryo showing the wild-type pattern of $\mathrm{S}$ phases. wglacZ expression is detected by the blue staining. (F) BrdU

incorporation in a homozygous DmcycE $E^{12 L}$ embryo showing $\mathrm{S}$ phases in the CNS, but fewer $S$ phases in the PNS and no $S$ phases in the epidermal thoracic patches. (G-H') Late stage11/early stage- 12 embryos. (G) $D m c y c E^{+}$sibling of the DmcycE ${ }^{19 L}$ embryo in $\mathrm{H}$, showing the wildtype pattern of cyclin E expression. The brown stain is the result of immunohistochemical staining of $\beta$-galactosidase derived from the balancer chromosome. $\left(\mathrm{G}^{\prime}\right)$ Higher magnification of thoracic patch expression in $\mathrm{G}$, showing the epithelial nature of these cells. (H) Homozygous DmcycE ${ }^{19 L}$ embryo showing transcripts in the CNS and PNS, but fewer cells in the epidermal thoracic patches expressing DmcycE than in G, most obviously in the first thoracic segment. $\left(\mathrm{H}^{\prime}\right)$ Higher magnification of thoracic patch expression in $\mathrm{H}$ showing the epithelial nature of these cells. (I,J) Early stage-12 embryos.

(I) $D m c y c E^{+}$sibling of the $D m c y c E^{19 L}$ embryo in $\mathrm{J}$, showing the wild-type pattern of cyclin $\mathrm{E}$ expression. The brown stain is the result of immunohistochemical staining of $\beta$-galactosidase derived from the balancer chromosome.

(J) Homozygous DmcycE ${ }^{19 L}$ embryos showing transcripts in the CNS and PNS. cns, central nervous system staining; pns, peripheral nervous system staining; mx, maxillary staining; lb, labial PNS staining; tp, thoracic patches.
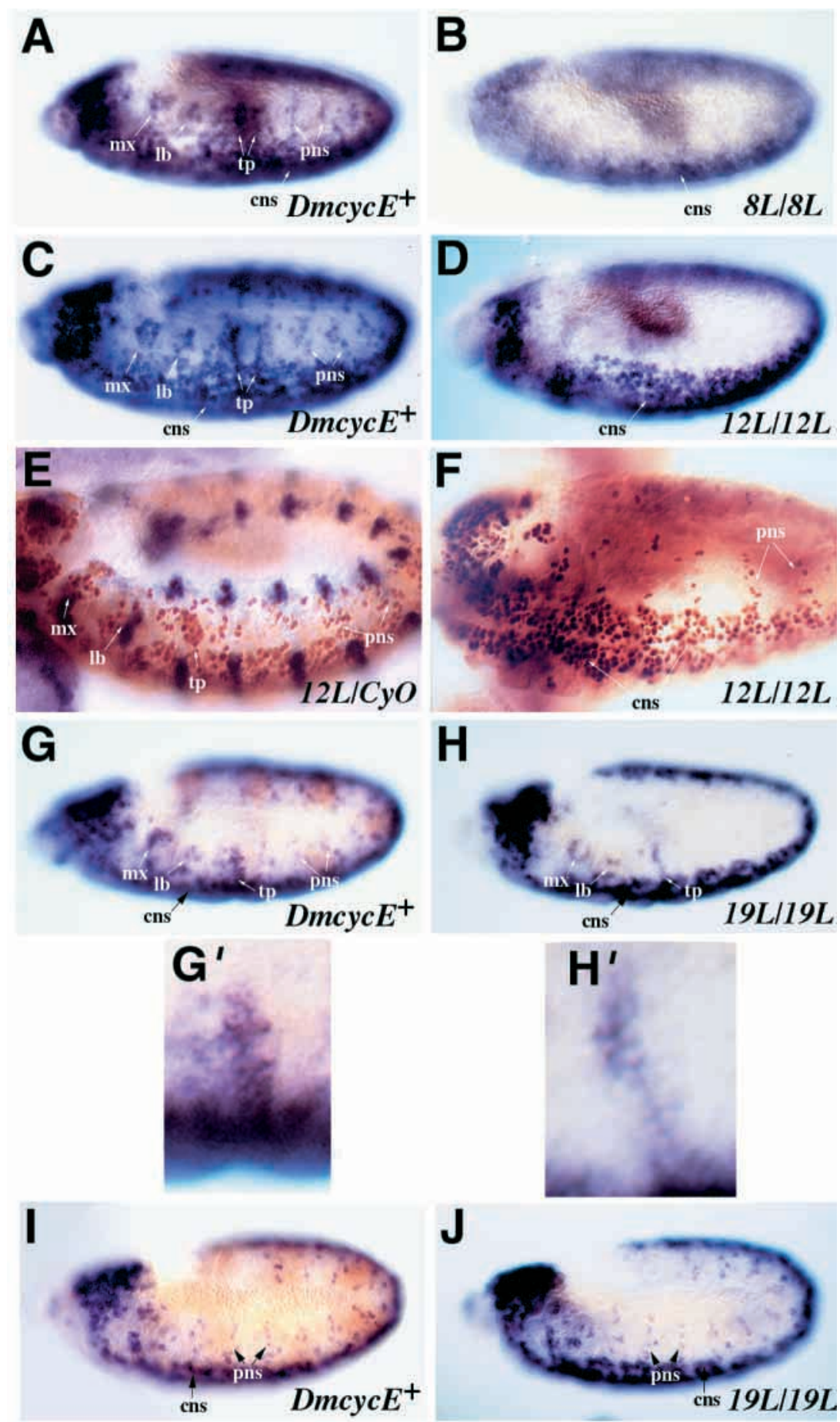

the distribution of DmcycE transcripts in homozygous DmcycE $E^{12 L}$ embryos (Fig. 3E,F).

Characterisation of embryos homozygous for DmcycE $E^{19 L}$, one of the smallest of the deletions (Fig. 1), revealed the presence of DmcycE transcripts in the CNS and PNS in a pattern similar to wild-type embryos, although the level of $D m c y c E$ transcripts in the PNS cells initially appeared to be less than in heterozygous sibling embryos (Fig. 3H,J, compare with G,I; data not shown). Interestingly, the DmcycE ${ }^{19 L}$ deletion partially disrupted DmcycE expression in the epidermal thoracic patches, as only a subset of the thoracic patch cells of the first thoracic segment expressed DmcycE (Fig. 3G, $\mathrm{G}^{\prime}$, compare with $\mathrm{H}, \mathrm{H}^{\prime}$ ), suggesting that these patches of expression may constitute a complex domain of DmcycE regulation.

These analyses define the location of separable DmcycE regulatory elements. Elements essential for DmcycE transcription in the PNS lie between the $3^{\prime}$ breakpoints of the $D m c y c E^{19 L}$ and DmcycE $E^{12 L}$ deletions (see Fig. 1), although other PNS enhancers required for initial maximal expression lie outside of this region. This region also contains elements required for a subset of the expression pattern in the epidermal 

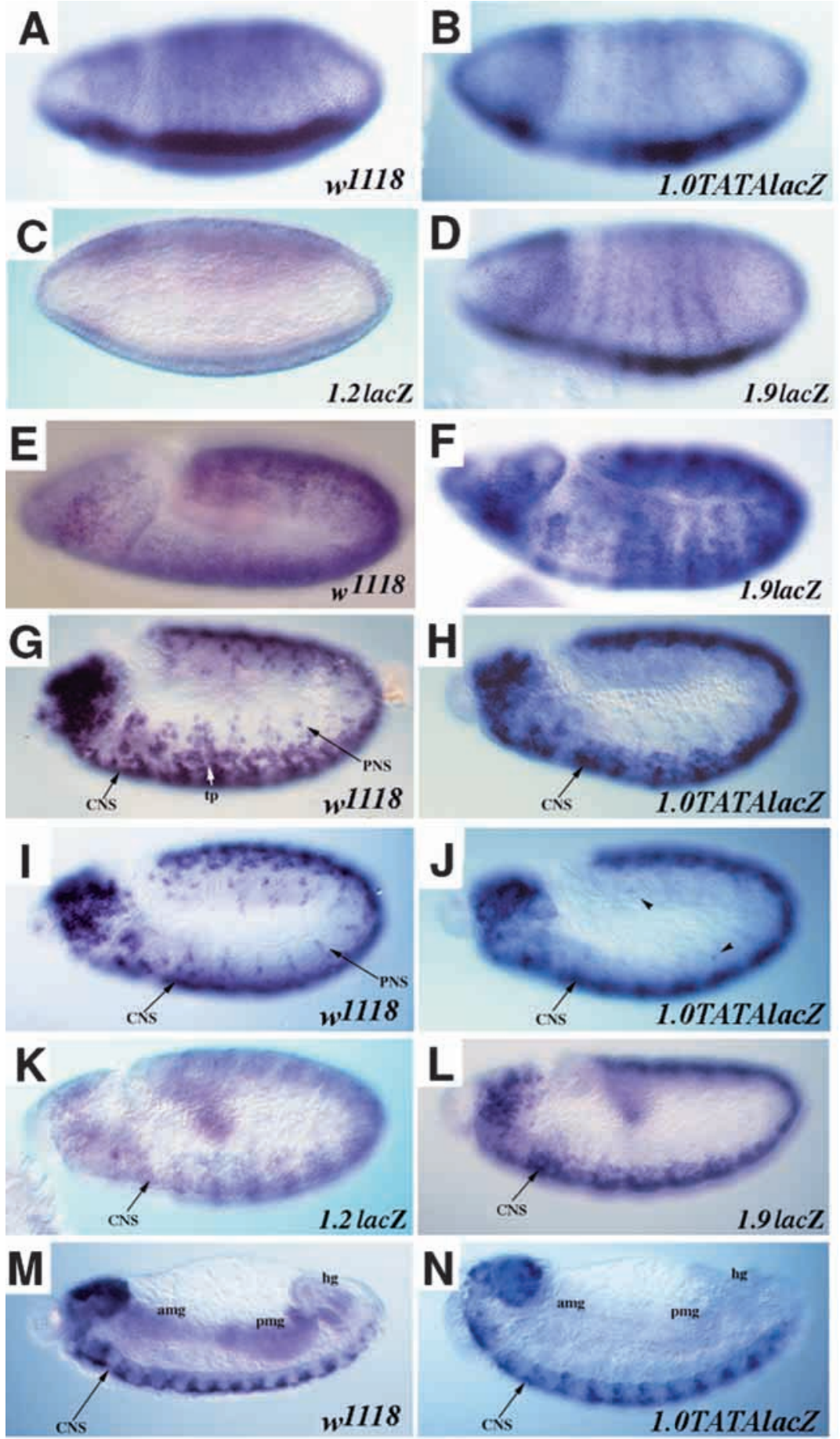

Fig. 4. DmcycE cis-acting elements that regulate expression in the CNS and epidermis during embryogenesis. Whole-mount in situ hybridisation using a DmcycE or $l a c Z$ reporter gene probe as indicated. (A-D) Stage 5-6 embryos. (A) $w^{1118}$ embryo, DmcycE probe; (B) 1.0TATAlacZ embryo, lacZ probe; (C) 1.2lacZ embryo, lacZ probe; (D) 1.9lacZ embryo, lacZ probe. (E,F) Late stage-9 embryos. (E) $w^{1118}$ embryo, DmcycE probe, showing general epidermal expression of DmcycE. (F) 1.9 lacZ embryo, lacZ probe, showing general epidermal expression of the lacZ reporter gene. $(\mathrm{G}, \mathrm{H})$ Stage-11 embryos. (G) $w^{1118}$ embryo, DmcycE probe;

(H) 1.OTATAlacZ embryo, lacZ probe. Note the lack of PNS and epidermal thoracic patch transcripts. (I-L) Late stage-11 embryos. (I) $w^{1118}$ embryo, $D m c y c E$ probe. Note that epidermal thoracic patch staining has gone by this stage; (J) 1.OTATAlacZ embryo, lacZ probe showing a few faintly stained PNS cells indicated by arrowheads; (K) 1.2lacZ embryo, lacZ probe; (L) 1.9lacZ embryo, lacZ probe.

(M,N) Stage-13 embryos. (M) $w^{1118}$ embryo, DmcycE probe; (N) 1.0TATAlacZ embryo, lacZ probe. Note the absence of transcripts in the endoreplicative gut. CNS, central nervous system; PNS, peripheral nervous system; tp, thoracic patch; amg, anterior midgut; pmg, posterior midgut; hg, hindgut.

\section{Reporter gene analysis further defines DmcycE tissue-specific cis-acting regulatory elements}

To confirm and extend the results obtained from the previous analyses, a series of DmcycE promoter-lacZ reporter gene constructs (summarised in Fig. 1) were generated and introduced into flies using $P$-element-mediated germline transformation. All but one of the lac Z reporter constructs contained the immediate endogenous zygotic promoter sequences and the entire DmcycE 5' untranslated region (UTR) fused to the ATG of an open reading frame encoding a nuclear targeted $\beta$-galactosidase. The endogenous DmcycE promoter was favoured over a heterologous minimal TATA promoter because zygotic DmcycE transcription is under the control of a TATA-less promoter (L. Jones, M. Silson, W. Winnall, R. Saint and H. E. Richardson, unpublished). The only reporter gene construct in which a heterologous promoter was used contained the promoter proximal $1.0 \mathrm{~kb}$ HindIII/EcoRI genomic

thoracic patches. Regulatory elements that are required for the complete pattern of DmcycE transcription in the epidermal thoracic patches are located between the $3^{\prime}$ breakpoints of the $D m c y c E^{19 L}$ deletion and a group of small $P$-element-mediated deletions represented by DmcycE ${ }^{7 L}$ in Fig. 1. Embryos that are homozygous for these small deletions have a wild-type DmcycE transcription pattern in the CNS, PNS and the epidermal thoracic patches (results not shown). The locations of regulatory elements defined by these deficiencies are consistent with the locations determined by the analysis of the $P\left[w^{+} T I\right]$ transgene. fragment joined to the $h s p 27$ TATA box and lacZ sequences (the 1.0TATAlacZ construct, Fig. 1). With one exception, embryos homozygous for at least three independent insertions of each transgene were characterised using whole-mount in situ hybridisation to determine the pattern of lacZ reporter gene transcription. The exception was the 16.4lacZ construct, where only one line was characterised. The activity of this transformant was assumed to be unaffected by local chromosome position effects, as the lac $Z$ expression patterns produced were consistent with those of other constructs. 
5.5 lacZ

$A$
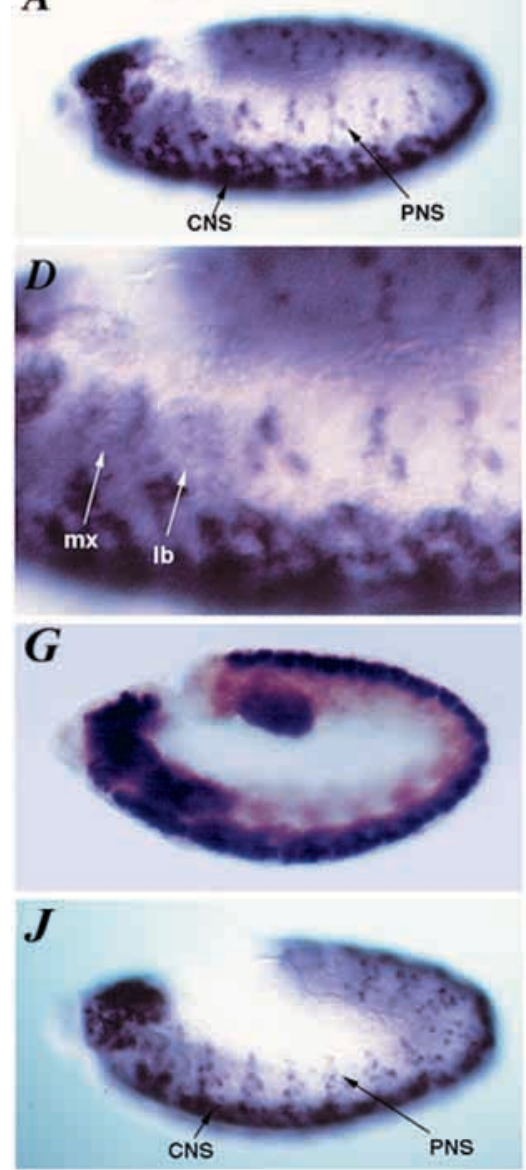

13.2 lacZ
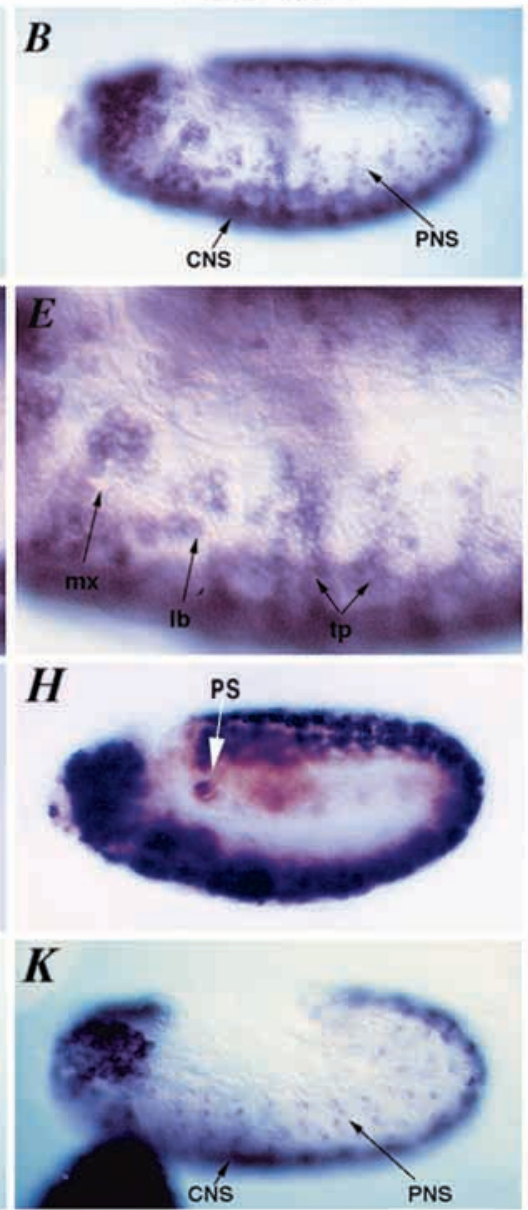

16.4 lacZ
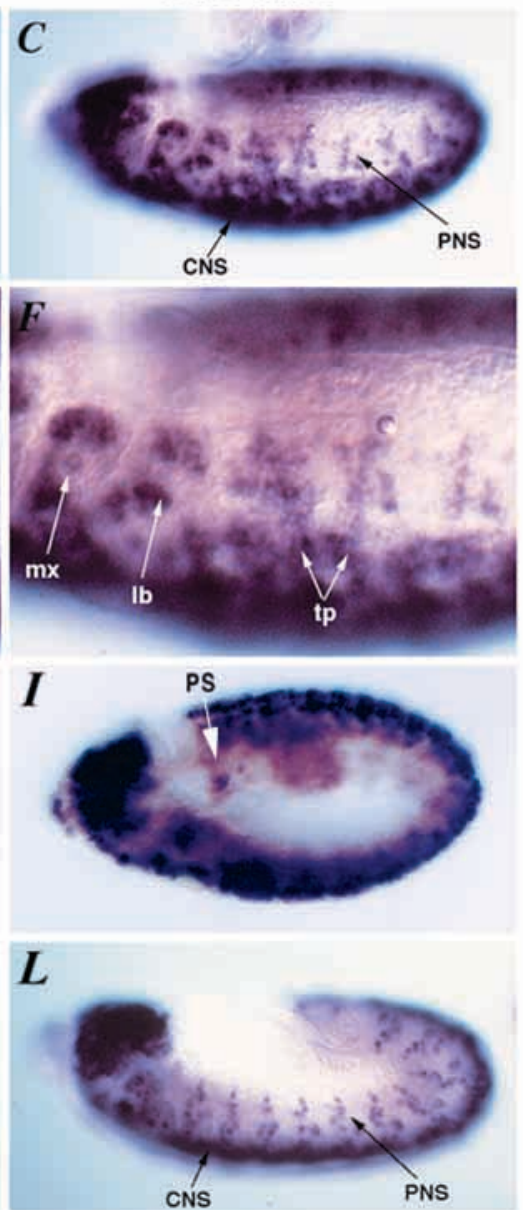

Fig. 5. DmcycE cis-acting elements that regulate expression in the PNS and epidermal thoracic patches. Whole-mount in situ hybridisation using a lacZ probe in stage-11 embryos (A-F). (A) 5.5lacZ embryo showing expression in the CNS and PNS cells; (B) 13.2lacZ embryo showing expression in the CNS and PNS cells; (C) 16.4lacZ embryo showing expression in the CNS and PNS cells; (D) higher magnification of the 5.5lacZ embryo shown in A. Expression is absent in the neuroblasts of the maxillary and labial segments as well as in the epidermal thoracic patches. (E) Higher magnification of the 13.2lacZ embryo shown in B, exhibiting expression in the CNS and PNS cells, including the neuroblasts of the maxillary and labial segments and expression in epidermal thoracic patch cells. (F) Higher magnification of the 16.4 lac $Z$ embryo shown in $\mathrm{C}$, exhibiting expression in the CNS and PNS cells, including the neuroblasts of the maxillary and labial segments and expression in epidermal thoracic patch cells. (G-I) Late stage-11 embryos. (G) 5.5lacZ embryo showing absence of expression in the posterior spiracle primordium; (H) 13.2lacZ embryo showing expression in the posterior spiracle primordium; (I) 16.4lacZ embryo showing expression in the posterior spiracle primordium. (J-L) Stage-12 embryos. (J) 5.5lacZ embryo showing expression in the CNS and PNS cells; (K) 13.2 lacZ embryo showing expression in the CNS and PNS cells; (L) 16.4lacZ embryo showing expression in the CNS and PNS cells. See Figs 2, 4 for abbreviations.

\section{CNS and early epidermal expression}

The 1.OTATAlacZ heterologous promoter construct and the $1.9 l a c Z$ endogenous promoter construct extend the same distance $5^{\prime}$ from the start of zygotic DmcycE transcription (Fig. 1). The patterns of lac $Z$ transcripts derived from these two constructs are essentially identical (Fig. 4 and data not shown), indicating that there was no obvious difference between the endogenous DmcycE and heterologous hsp27-TATA promoters.

Expression of the lac $Z$ reporter gene from the 1.0TATAlac $Z$ and 1.9 lac $Z$ endogenous promoter constructs was detected in the early epidermis (Fig. 4A,B,D-F) and also in the proliferating CNS (Fig. 4G-J,L-N) in a pattern similar to that normally seen for DmcycE. This indicates that cis-acting sequences required for driving $D m c y c E$ transcription in the epidermis during cycles 14-16 and in the CNS cells are located within this $1.0 \mathrm{~kb}$ fragment. Significantly, epidermal reporter gene expression was downregulated by the end of stage 11 (Fig. 4J,L, compare with G), suggesting that the sequences driving expression of the reporter constructs include the regulatory elements responsible for the downregulation of wild-type DmcycE.

In contrast to the normal pattern of epidermal and CNS expression from the 1.0TATAlac $Z$ and 1.9 lac $Z$ constructs, there was a dramatic absence of lac $Z$ transcripts in most of the proliferating PNS cells. Very low levels of lacZ expression were detected in one or two PNS cells per segment (Fig. 4G$\mathrm{J}, \mathrm{L})$. This result is consistent with analysis of the DmcycE $E^{12 L}$ regulatory region deletion (see above) and indicates that DmcycE transcription in the CNS and PNS cells is regulated by different $c i s$-acting sequences. In addition, lac $Z$ transcripts 


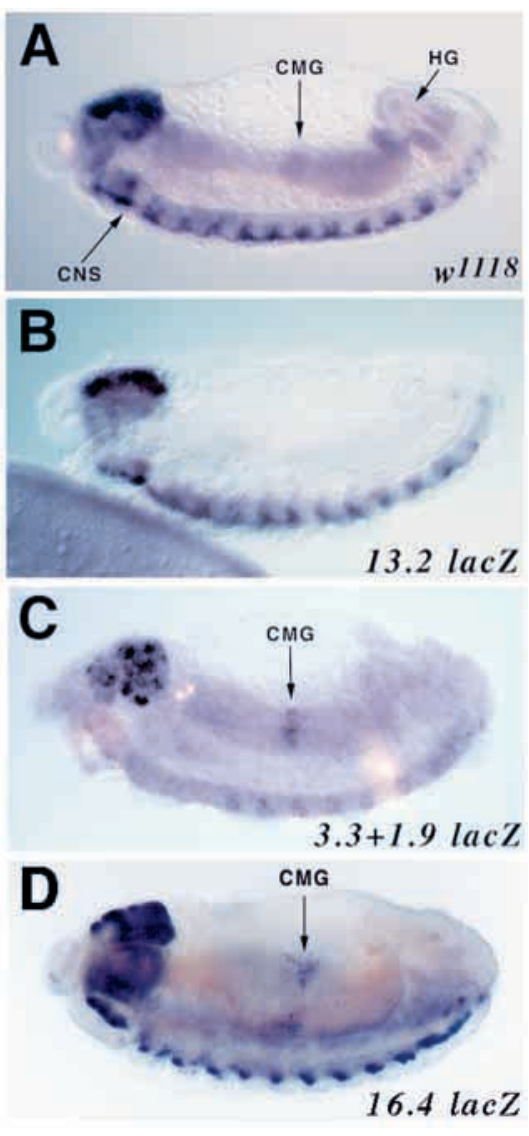

Fig. 6. DmcycE cis-acting elements that regulate expression in the endoreplicative gut. Whole-mount in situ hybridisation using a $D m c y c E$ or $l a c Z$ reporter gene probe as indicated, showing reporter gene expression in an endoreplication domain in the central midgut driven by sequences more than $12 \mathrm{~kb} 5^{\prime}$ of the transcription start site All embryos are shown at stage 13. (A) $w^{1118}$ embryo, DmcycE probe; (B) 13.2lacZ embryo, lacZ probe; (C) 3.3+1.9lacZ embryo, lac $Z$ probe; (D) 16.4 lac $Z$ embryo, lac $Z$ probe. $C M G$, central midgut; $\mathrm{HG}$, hindgut; CNS, central nervous system.

were not detected in the epidermal thoracic patches nor in endoreplicating tissues (Fig. 4G,H,M,N), suggesting that essential regulatory sequences controlling DmcycE transcription in these tissues are located outside of the $1.0 \mathrm{~kb}$ HindIII/EcoRI genomic fragment, again consistent with the phenotypic analysis of deficiencies and the genomic fragment described above (summarised in Fig. 7). Consistent with all necessary epidermal and CNS elements being localised to the $1.0 \mathrm{~kb}$ HindIII/EcoRI fragment, all larger constructs containing this region exhibited expression of lac $Z$ in the epidermis during mitotic cycles 14-16 and in the proliferating CNS cells (data not shown).

Embryos homozygous for the 1.2lacZ construct, which contains approximately the last $400 \mathrm{bp}$ of the $1.0 \mathrm{~kb}$ HindIII/EcoRI fragment, expressed lac $Z$ at a very low level in both the epidermis and the CNS (Fig. 4C,K). This suggests that the enhancers necessary for this pattern of DmcycE transcription are located in this $400 \mathrm{bp}$ fragment. However, as the level of lac $Z$ transcripts is markedly reduced relative to the 1.0TATAlac $Z$ and $1.9 l a c Z$ constructs, regulatory elements that increase the level of transcription in this pattern must be located in the remaining $600 \mathrm{bp}$ region of the $1.0 \mathrm{~kb}$ HindIII/EcoRI fragment.

\section{PNS expression}

The 5.5lac Z construct is the smallest endogenous promoter construct that gave a pattern of lacZ expression in PNS cells similar to that observed for wild-type DmcycE (Fig. 5A,D,J). Interestingly, the pattern of PNS lacZ transcripts was not complete as it lacked expression in PNS neuroblasts of the maxillary and labial segments (Fig. 5D). This result is consistent with the pattern of zygotic DmcycE transcription

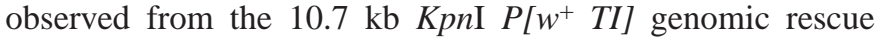
construct, which extends to the same $5^{\prime} K p n I$ restriction enzyme site used in the 5.5lac $Z$ construct. The next smallest endogenous promoter construct, the 2.9lacZ, fails to express lacZ in the PNS in all but 1 or 2 cells per segment (data not shown), so the regulatory sequences responsible for driving DmcycE transcription in proliferating thoracic and abdominal PNS cells must be located in a $2.6 \mathrm{~kb} \mathrm{KpnI/NcoI}$ genomic fragment (summarised in Fig. 7).

Embryos homozygous for either the 13.2lacZ or the 16.4lacZ endogenous promoter constructs showed lac Z transcripts in all of the proliferating PNS cells (Fig. 5B,C,E,F,K,L). This pattern was indistinguishable from the wild-type DmcycE PNS expression pattern, since lacZ transcripts were now present in the maxillary and labial segment PNS neuroblasts (Fig. 5B,C,E,F). The detection of lacZ PNS transcripts in the maxillary and labial head segments from the 13.2lacZ and 16.4lacZ constructs and the absence of this pattern from the $5.57 a c Z$ construct indicate that enhancer sequences necessary for $D m c y c E$ transcription in the maxillary and labial segment PNS cells are located in the $7.7 \mathrm{~kb}$ NheI/KpnI genomic fragment (summarised in Fig. 7).

\section{Expression in cycle 17 thoracic epidermal cells}

lacZ transcripts in the epidermal thoracic patches were observed in embryos homozygous for the 13.2lac $Z$ and 16.4lacZ promoter constructs (Fig. 5B,C,E,F). The pattern is similar to wild-type $D m c y c E$ transcription in the thoracic patches in the epidermis of the first and second thoracic segments. DmcycE expression can also be observed in a small patch in the ventral epidermis of the third thoracic segment, but this expression occurs in a much smaller group of cells and more transiently than in the first and second thoracic patches. As a result it was very difficult to observe and was not analysed in detail. As lacZ transcripts in the epidermal thoracic patches were not observed from embryos homozygous for the 5.5lacZ construct (Fig. 5A,D), sequences required for driving DmcycE transcription in the epidermal thoracic patches must be located within the $7.7 \mathrm{~kb}$ NheI/KpnI fragment (see Fig. 7). The location of this cis-acting regulatory element is consistent with the analysis of DmcycE transcription in embryos homozygous for the $D m c y c E^{12 L}$ regulatory region deletion, which removes this

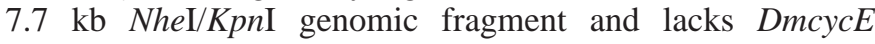
transcription in the epidermal thoracic patches (see above).

\section{Expression in the posterior spiracle primordia}

Stage-11 and -12 embryos express $R n r 2$ in the posterior spiracle primordia prior to $\mathrm{S}$ phase entry at this stage (Duronio and O'Farrell, 1995). DmcycE is also expressed in these cells (data not shown). LacZ transcripts in the posterior spiracle 


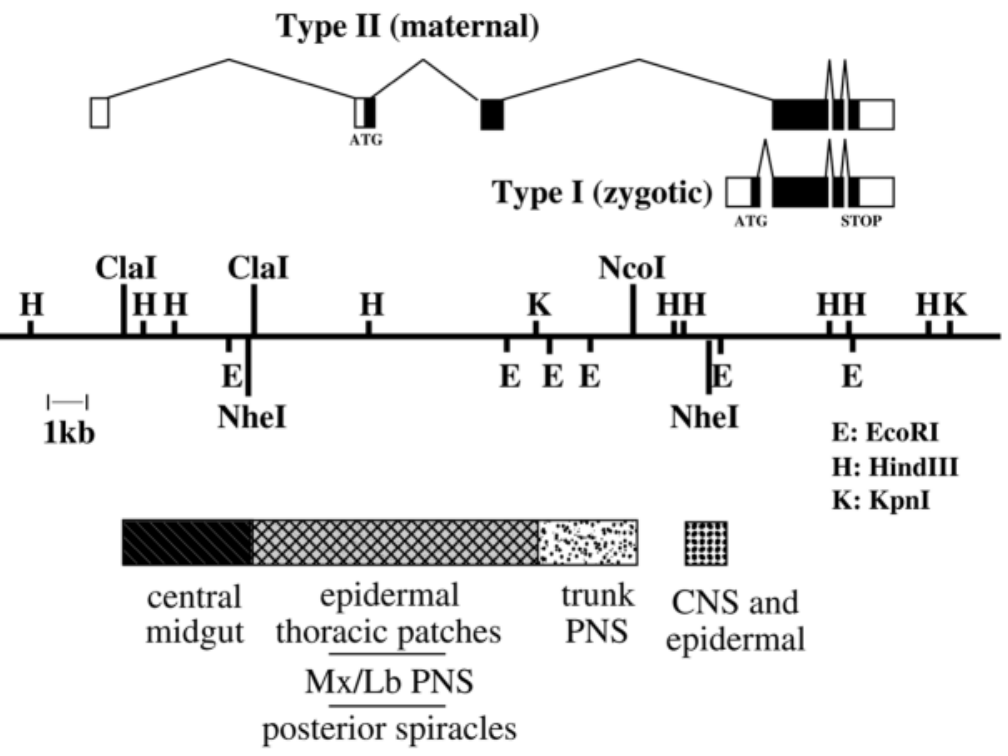

primordia were observed in embryos carrying the 13.2 lac $Z$ and 16.4lacZ constructs (Fig. 5H,I), but not in embryos carrying the 5.5lac Z construct (Fig. 5G). This indicates that the regulatory region responsible for DmcycE expression in the posterior spiracle primordia is located within the $7.7 \mathrm{~kb}$ NheI/KpnI fragment, the same region that contains the regulatory sequences required for expression in the epidermal thoracic patches.

\section{Expression in endoreplicating tissues}

Analysis of the series of DmcycE promoter-lacZ reporter constructs, up to and including the $13.2 \mathrm{lac} Z$ construct, failed to identify the location of any regulatory elements responsible for driving DmcycE transcription in the endoreplicating tissues (Figs 4N, 6B; data not shown), indicating that such elements are located outside of the $13.2 \mathrm{~kb} N h e \mathrm{I} / \mathrm{NcoI}$ region covered in this analysis. This conclusion is consistent with our observations that the $10.7 \mathrm{~kb} K p n \mathrm{I} P[w+T I]$ rescue construct exhibits no detectable $D m c y c E$ expression in endoreplicating cells (see Fig. 2G).

Interestingly, analysis of two DmcycE promoter-lacZ reporter constructs has identified a $3.3 \mathrm{~kb}$ ClaI genomic fragment that contains regulatory elements important for transcription in a subset of the endoreplicating midgut. Embryos homozygous for either the 16.4lacZ construct or the $3.3+1.9 l a c Z$ (see Fig. 1), show a low level of lacZ expression in cells of the embryonic central midgut (Fig. 6C,D), which is not present in embryos containing the 13.2lac Z construct (Fig. $6 \mathrm{~B})$. However, regulatory elements that are required for $D m c y c E$ transcription in the anterior and posterior midgut, hindgut, salivary glands and Malpighian tubules remain to be identified.

\section{DISCUSSION}

We have previously shown that regulated $D m c y c E$ transcription is required to maintain the normal pattern of $S$ phases during Drosophila development (Knoblich et al., 1994; Richardson et al., 1995; Secombe et al., 1998). However, the mechanism by

which developmental signals and patterning cues are integrated to coordinate $D m c y c E$ transcription could occur in many ways. For example, DmcycE could be regulated in a relatively simple manner, with multiple developmental signals inducing the expression of a common trans-acting factor that acts on a small number of DmcycE regulatory elements. Alternatively, developmental regulation of $D m c y c E$ transcription may be more direct, with a complex set of DmcycE cis-regulatory elements being responsible for integrating developmental signals and patterning cues.

The data presented in this study supports the second hypothesis. Our analyses revealed the presence of multiple independent tissue-specific regulatory elements acting to control DmcycE transcription during embryogenesis. These include (1) at least two different elements required for expression in different cells of the peripheral nervous system, (2) an element required for expression in the epidermal cells and central nervous system and (3) an element required for expression in patches of thoracic epidermal cells that undergo a 17th $\mathrm{G}_{1}$ phase-regulated cycle, in the PNS of the labial and maxillary segments and in the posterior spiracle primordia. These elements were identified by the presence or absence of tissue-specific expression in a genomic transformant, in animals carrying small regulatory region deletions generated by $P$-element excisions and in genomic fragment-reporter gene constructs. The results obtained with each of these different approaches were consistent with the others in terms of the location of the different elements. One notable group of elements that were not fully defined in this study are those responsible for DmcycE expression in endoreplicating tissues. Only one element was identified that was responsible for driving DmcycE transcription in the central midgut. Presumably elements responsible for regulating the remaining patterns of DmcycE in embryonic endoreplicating tissues lie further $5^{\prime}$ or $3^{\prime}$ of the genomic regions examined in this study. Our failure to define more than the one endoreplicative regulatory sequence is surprising. It is possible that other regulatory sequences lie outside the region studied, but it is also possible that identification of these domains may be hampered by the negative autoregulatory nature of $D m c y c E$ expression in 
these endoreplication domains (Duronio and O'Farrell, 1995; Sauer et al., 1995). If the elements carrying the endoreplication regulatory sequences also carry the autorepression sequences, expression may be inhibited by the endogenous DmcycE. It should also be noted that additional complexity is likely to exist in the regulation of DmcycE transcription. For example, $D m c y c E$ was expressed in a subset of the epidermal thoracic patch cells in $D m c y c E^{19 L}$ deficient embryos, indicating that these patches may themselves be complex domains of DmcycE transcriptional regulation.

Although our analyses have identified separable tissuespecific cis-acting elements in the DmcycE regulatory region, we have yet to elucidate the factors that operate on these elements to drive DmcycE transcription. Some clues to the trans-acting factors that could be regulating DmcycE transcription come from studies of cell cycle control mechanisms in mammalian cells, where cyclin $E$ transcriptional regulation also plays an important role in control of the $\mathrm{G}_{1}$ to $\mathrm{S}$ phase transition (Ohtsubo and Roberts, 1993; Resnitzky et al., 1994). A well-established model of $\mathrm{G}_{1}$ phase transcriptional regulation in mammalian cells postulates a cascade of events initiated by extracellular growth factor signalling that leads to activation of the Cyclin D/Cdk4 complex, which in turn phosphorylates the tumor suppressor Retinoblastoma $(\mathrm{Rb})$, disassociating it from the $\mathrm{S}$ phasespecific transcription factors E2F and DP and allowing transcription of $\mathrm{S}$ phase-specific genes, such as cyclin $E$ (reviewed in Dyson, 1998).

In Drosophila, where we have an opportunity to examine $\mathrm{G}_{1}$ phase-regulatory events within a developmental context, $d E 2 F$ and $d D P$ are required for embryonic DmcycE transcription in endoreplicating tissues, but are dispensable for DmcycE transcription in the CNS divisions that lack $\mathrm{G}_{1}$ and G2 phases (Duronio and O'Farrell, 1995; Royzman et al., 1997; Duronio et al., 1998). It is not known whether $d E 2 F$ and $d D P$ are required for $D m c y c E$ expression in the early embryonic PNS and epidermal divisions, because maternal products of these genes may mask any requirement. Nonetheless, the fact that these transcription factors are dispensable for DmcycE transcription in the CNS cells argues against the universality of the mammalian model of cyclin $E$ regulation by E2F. In addition, the mammalian model predicts that E2F-dependent transcriptional regulation of $D m c y c E$ in endoreplicating tissues should be mediated through an E2F/DP responsive element. In the experiments described here, we have shown that this model is an oversimplification of DmcycE regulation in Drosophila, as only one element in the $16 \mathrm{~kb}$ analysed was found to drive $D m c y c E$ expression in a subset of endoreplicating tissues. Thus a minimum of two regulatory elements are required for driving $D m c y c E$ expression in endoreplicating tissues. The fact that expression of DmcycE in these tissues has been shown to be dependent on the E2F/DP complex appears at first sight to be paradoxical. The paradox would be resolved if expression requires activation by both E2F/DP and tissue-specific activators. An absolute requirement for a developmental signal cannot however exist, as ectopic expression of E2F and DP together induces DmcycE expression in all $\mathrm{G}_{1}$-arrested epidermal cells (Duronio et al., 1996). It remains possible that the high levels of E2F and DP expressed following heat shock induction of the respective transgenes overrides the tissuespecific regulatory component.
Downregulation of DmcycE transcription during cycle 16 is essential for cycle $17 \mathrm{G}_{1}$ arrest in epidermal cells prior to differentiation (Knoblich et al., 1994). Significantly, the regulatory element that drives constitutive $D m c y c E$ expression in the epidermal cells during cycles 14-16 showed transcriptional downregulation, characteristic of wild-type $D m c y c E$ expression. If this downregulation requires active repression of DmcycE transcription, then the regulatory sequences necessary for this repression must also be located in the $1.0 \mathrm{~kb}$ regulatory element defined here. Alternatively, the downregulation could be a consequence of the loss of activation of DmcycE transcription in the epidermis. Interestingly, a study by Du and Dyson (1999), revealed that $D m c y c E$ is ectopically expressed in a subset of normally $\mathrm{G}_{1}$ arrested epidermal cells in embryos that are deficient for $R B F$ (the Drosophila Rb homolog). However, DmcycE transcription is initially downregulated normally and a $\mathrm{G}_{1}$ cycle 17 arrest is established in the absence of RBF. These data suggest that $D m c y c E$ is actively repressed in $\mathrm{G}_{1}$-arrested epidermal cells and that $\mathrm{RBF}$ is required to maintain this repression. Although $d E 2 F$ or $d D P$ do not result in ectopic $D m c y c E$ transcription in epidermal cells (Duronio and O'Farrell, 1995; Royzman et al., 1997; Duronio et al., 1998) a RBF/E2F/DP complex may still mediate this repression if maternal E2F and DP are not depleted in the respective mutant embryos at this stage. A transcriptional repression mechanism of DmcycE at this stage may also be mediated by the second Drosophila E2F, E2F2, which has been shown to act as a transcriptional repressor of $\mathrm{S}$ phase genes in tissue culture cells (Sawado et al., 1998). Alternatively, E2F and/or DP may be needed to activate ectopic DmcycE expression in the absence of RBF. The initial mechanism acting to downregulate DmcycE transcription remains to be determined. Further dissection of this regulatory element may identify separate activation and repression sequences.

The regulators of DmcycE transcription in other developmental contexts may be more difficult to identify, as many candidate genes may be involved. In some cases it is possible to suggest the involvement of particular regulatory genes. For example, the discrete expression of $D m c y c E$ in the epidermal thoracic patches, which undergo a $\mathrm{G}_{1}$-regulated 17 th cell cycle, is much stronger in the first thoracic segment and is absent in the abdominal segments. The products of the homeotic genes of the Bithorax and Antennapedia Complexes, which are key components of anterior/posterior patterning (Heuer and Kaufman, 1992; Castelli-Gair and Akam, 1995) are therefore candidate upstream regulators of DmcycE transcription in this tissue.

\section{Complex transcriptional regulation of cell cycle regulatory genes: a common theme?}

Our understanding of cell cycle regulation has primarily derived from the single cell yeast, from cultured cells and from oocytes or very early embryos in which the complex patterning of embryogenesis has not yet begun. In contrast, cell cycles in the embryos of a multicellular organism respond to a variety of developmental cues that give different tissue types and different cell cycle kinetics. The significance of the relationship between embryo patterning and cell cycle control is evident from the pioneering work of Edgar and O'Farrell (1989, 1990), in which regulation of the cycles that occur during gastrulation 
and germ band extension in Drosophila embryos was shown to be mediated by the transcriptional regulation of the string (stg) mitotic activator gene. This transcriptional regulation is mediated by the patterning genes active during these stages.

The stg mitotic inducer gene exhibits a remarkably complex transcriptional regulatory region that responds to a variety of patterning genes to control cell cycle progression during early embryogenesis (Edgar et al., 1994; Lehman et al., 1998). The analysis of DmcycE regulation presented here offers an interesting parallel with stg in that both genes exhibit unexpected complexity in transcriptional regulation. It is striking that both $D m c y c E$ and $s t g$, the first cell cycle regulators to have their transcriptional regulation examined in a developmental context, contain tissue-specific regulatory elements. This indicates that transcriptional regulation is mediated differently in distinct developmental contexts, such as during the complex events of gastrulation when cycles 1416 occur or during the 17th cycle in cells of the epidermal thoracic patches.

Why is DmcycE transcriptional regulation so complex? One clue comes from the observation that the $\mathrm{G}_{1}$ regulators cyclin $D$, cyclin $E, R b$ and $E 2 F / D P$ are highly conserved between insects and mammals, but have no specific orthologs in yeast (Reed, 1996). Perhaps these genes represent a specialised mechanism that evolved to deal with $G_{1}$ phase regulation in multicellular organisms. Complex transcriptional regulation of cell cycle genes such as DmcycE may be part of such a mechanism, which permitted the universal eukaryotic cell cycle regulatory genes to be brought under the influence of the more recently evolved transcriptional regulatory mechanisms operating in different tissues and at different developmental stages.

We acknowledge the technical assistance of Julianne Camerotto in generating the Type I genomic $P[w+T 1]$ transgenic flies and Angela Gardiakos for help with BrdU incorporation. We thank Bob Duronio for discussions of work prior to publication. L. J. was supported by an Australian Post Graduate Award. This work was supported by grants from the National Health and Medical Research Council of Australia and the Australian Research Council.

\section{REFERENCES}

Bate, M. and Martinez Arias, A. (1991). The embryonic origin of imaginal discs in Drosophila. Development 112, 755-761.

Castelli-Gair, J. and Akam, M. (1995). How the Hox gene Ultrabithorax specifies two different segments, the significance of spatial and temporal regulation within metameres. Development 121, 2973-2982.

Du, W. and Dyson, N. (1999). The role of RBF in the introduction of G1 regulation during Drosophila embryogenesis. EMBO J. 18, 916-925.

Duronio, R. J. and O'Farrell, P. H. (1995). Developmental control of the G1 to $\mathrm{S}$ transition in Drosophila, cyclin E is a limiting downstream target of E2F. Genes Dev. 9, 1456-1468.

Duronio, R. J., Brook, A., Dyson, N. and O'Farrell, P. H. (1996). E2Finduced S phase requires cyclin E. Genes Dev. 10, 2505-2513.

Duronio, R. J., Bonnette, P. C. and O'Farrell, P. H. (1998). Mutations of the Drosophila $d D P, d E 2 F$ and cyclin $E$ genes reveal distinct roles for the E2F-DP transcription factor and cyclin E during G1-S transition. Mol. Cell. Biol. 18, 141-151.

Dyson, N. (1998). The regulation of E2F by pRB-family proteins. Genes Dev. 12, 2245-2262.

Edgar, B. A. and O'Farrell, P. H. (1989). Genetic control of cell division patterns in the Drosophila embryo. Cell 57, 177-187.

Edgar, B. A. and O'Farrell, P. H. (1990). The three postblastoderm cell cycles of Drosophila embryogenesis are regulated in G2 by string. Cell $\mathbf{6 2}$, 469-480.

Edgar, B. A., Lehman, D. A. and O'Farrell, P. H. (1994). Transcriptional regulation of string ( $c d c 25)$, a link between developmental programming and the cell cycle. Development 120, 3131-3143.

Foe, V. E. (1989). Mitotic domains reveal early commitment of cells in Drosophila embryos. Development 107, 1-27.

Follette, P. J. and O'Farrell, P. H. (1997). Cdks and the Drosophila cell cycle. Curr. Opin. Genet. Dev. 7, 17-22.

Follette, P. J., Duronio R. J. and O'Farrell P. H. (1998). Fluctuations in cyclin $\mathrm{E}$ levels are required for multiple rounds of endocycle $\mathrm{S}$ phase in Drosophila. Curr. Biol. 8, 235-238.

Go, M. J., Eastman, D. S. and Artavanis-Tsakonas, S. (1998). Cell proliferation control by Notch signaling in Drosophila development. Development 125, 2031-2040.

Hartenstein, V., Rudloff, E. and Campos-Ortega, J. A. (1987). The pattern of proliferation of the neuroblasts in the wild-type embryo if Drosophila melanogaster. Wilhelm Roux's Arch. Dev. Biol. 196, 473-485.

Heuer, J. G. and Kaufman, T. C. (1992). Homeotic genes have specific functional roles in the establishment of the Drosophila embryonic peripheral nervous system. Development 115, 35-47.

Horsfield, J., Penton, A., Secombe, J., Hoffman, F. M. and Richardson, H. (1998). decapentaplegic is required for arrest in G1 phase during Drosophila eye development. Development. 125, 5069-5078.

Johnston, L. A. and Edgar, B. A. (1998). Wingless and Notch regulate cellcycle arrest in the developing Drosophila wing. Nature 394, 82-84.

Knoblich, J. A., Sauer, K., Jones, L., Richardson, H., Saint, R. and Lehner, C. F. (1994). Cyclin E controls S phase progression and its downregulation during Drosophila embryogenesis is required for the arrest of cell proliferation. Cell 77, 107-120.

Lehman, D. A., Patterson, B., Johnston, L. A., Balzer, T., Britton, J. S., Saint, R. and Edgar, B. A. (1998). Cis-regulatory elements of the mitotic regulator, string/Cdc25. Development 126, 1793-1803.

Li, L. and Vaessin, H. (2000). Pan-neural Prospero terminates cell proliferation during Drosophila neurogenesis. Genes Dev. 14, 147-151.

Masucci, J. D., Miltenberger, R. J. and Hoffmann, F. M. (1990). Patternspecific expression of the Drosophila decapentaplegic gene in imaginal disks is regulated by $3^{\prime}$ cis-regulatory sequences. Genes Dev. 4, 20112023.

Myster, D. L. and Duronio, R. J. (2000). Cell cycle, to differentiate or not to differentiate? Curr. Biol. 10, R302-304.

Ohtsubo, M. and Roberts, J. M. (1993). Cyclin-dependent regulation of G1 in mammalian fibroblasts. Science 259, 1908-1912.

Ohtsubo, M., Theodoras, A. M., Schumacher, J., Roberts, J. M. and Pagano, M. (1995). Human Cyclin E, a nuclear protein essential for the G1to-S phase transition. Mol. Cell. Biol. 15, 2612-2624.

Pines, J. and Hunter, T. (1991). Cyclin-dependent kinases, a new cell cycle motif? Trends Cell Biol. 1, 117-121.

Reed, S. I. (1992). The role of p34 kinases in the G1 to S phase transition. Ann. Rev. Cell Biol. 8, 529-561.

Reed, S. I. (1996). G1/S regulatory mechanisms from yeast to man. In Progress in Cell Cycle Research, Vol. 2 (ed. L. Meijer, S. Guidet and L. Vogel), pp. 15-27. New York: Plenum Press.

Resnitzky, D., Gossen, M., Bujard, H. and Reed, S. I. (1994). Acceleration of the G1/S transition by expression of cyclins D1 and E using an inducible system. Mol. Cell. Biol. 14, 1669-1679.

Richardson, H. E., O'Keefe, L. V. Reed, S. I. and Saint, R. (1993). A Drosophila G1-specific cyclin E homolog exhibits different modes of expression during embryogenesis. Development 119, 673-690.

Richardson, H. E., O'Keefe, L. V., Marty, T. and Saint, R. (1995). Ectopic cyclin $\mathrm{E}$ expression induces premature entry into $\mathrm{S}$ phase and disrupts pattern formation in the Drosophila eye imaginal disc. Development 121, 3371-3379.

Richter, S., Hartmann, B. and Reichert, H. (1998). The wingless gene is required for embryonic brain development in Drosophila. Dev. Genes Evol. 208, 37-45.

Royzman, I., Whittaker, A. J. and Orr-Weaver, T. L. (1997). Mutations in Drosophila DP and E2F distinguish G1-S progression from an associated transcriptional program. Genes Dev. 11, 1999-2011.

Sauer, K., Knoblich, J. A., Richardson, H. and Lehner, C. F. (1995). Distinct modes of cyclin E/cdc2c kinase regulation and S-phase control in mitotic and endoreduplication cycles of Drosophila embryogenesis. Genes Dev. 9, 1327-1339.

Sawado, T., Yamaguchi, M., Nishimoto, Y., Ohno, K., Sakaguchi, K. and 
Matsukage, A. (1998). dE2F2, a novel E2F-family transcription factor in Drosophila melanogaster. Biochem Biophys Res Commun. 251, 409-415.

Secombe, J., Pispa J., Saint, R. and Richardson, H. (1998). Analysis of a Drosophila cyclin $E$ hypomorphic mutation suggests a novel role for Cyclin $\mathrm{E}$ in cell proliferation control during eye imaginal disc development. Genetics 149, 1867-1882.

Serrano, N. and O'Farrell, P. H. (1997). Limb morphogenesis, connections between patterning and growth. Curr. Biol. 7, 186-195.

Skaer, H. and Martinez-Arias, A. (1992). The wingless product is required for cell proliferation in the Malpighian tubule anlage of Drosophila melanogaster. Development 116, 745-754.

Smith, A. V. and Orr-Weaver, T. L. (1991). The regulation of the cell cycle during Drosophila embryogenesis, the transition to polyteny. Development 112, 997-1008.

Tautz, D. and Pfeifle, C. (1989). A non-radioactive in situ hybridization method for the localization of specific RNAs in Drosophila embryos reveals translational control of the segmentation gene hunchback. Chromosoma $\mathbf{9 8}$, $81-85$.

Weigmann, K. and Lehner, C. F. (1995). Cell fate specification by evenskipped expression in the Drosophila nervous system is coupled to cell cycle progression. Development 121, 3713-3721.

Weiss, A., Herzig, A., Jacobs, H. and Lehner, C. F. (1998). Continuous Cyclin E expression inhibits progression through endoreduplication cycles in Drosophila. Curr. Biol. 8, 239-242. 